\title{
Central exclusive photoproduction at the LHC
}

\author{
Wolfgang SCHÄFER ${ }^{*}$ \\ IFJ PAN, Kraków \\ E-mail: wolfgang.schaferdifj.edu.pl \\ Anna CISEK \\ IFJ PAN, Kraków \\ E-mail: anna.cisek@ifj.edu.pl \\ Antoni SZCZUREK \\ IFJ PAN, Kraków and Rzeszów University \\ E-mail: antoni.szczurek@ifj.edu.pl
}

\begin{abstract}
Protons and antiprotons at collider energies are a source of high energy Weizsäcker-Williams photons. This opens up a possibility to study at the LHC exclusive photoproduction of heavy vector mesons at energies much larger than possible at the HERA accelerator. We present selected results of our detailed studies of various distributions for the production of heavy quarkonia (e.g. rapidity, transverse momenta, azimuthal angles). We give predictions for LHC energies. Our calculations are based on modelling the photoproduction amplitudes in a $k_{\perp}$-factorisation approach which is checked against HERA data. We also discuss the exclusive photoproduction of $Z^{0}$ bosons.
\end{abstract}

European Physical Society Europhysics Conference on High Energy Physics

July 16-22, 2009

Krakow, Poland

\footnotetext{
* Speaker.

${ }^{\dagger}$ Partially supported by the Polish Ministry of Science and Higher Education under contract 1916/B/H03/2008/34.
} 
Central exclusive production processes have recently attracted much attention for offering a rich physics program at the modern colliders, from a potentially clean way to discover/investigate a light Higgs to (exotic) hadron spectroscopy to investigations of the QCD Pomeron (for recent reviews with many references, see [1] $)$.

Here we show a selection of results obtained in [2, 3, 他. We concentrate on a particular production mechanism: photoproduction. In the language of $t$-channel exchanges, we describe it in terms of the photon-Pomeron fusion. We want to have large rapidity gaps, so the exchange mechanism must have an effective spin $\geq 1$, and must not carry neither charge nor color charge. Those requirements are fulfilled by both photon and Pomeron. The $C$-parity of the centrally produced system must be the product $C=C_{\gamma} \cdot C_{\mathbf{P}}=-1$. Hence we will concentrate on the production of (heavy) vector mesons $J / \psi, \Upsilon$ and their radial excitations, and we will also show predictions for the electroweak $Z^{0}$ gauge boson.

Ideally one would measure the final state completely, including the protons. An interesting feature of the photon exchange mechanism is that the interference between the photon-Pomeron fusion, and the exchange-diagram Pomeron-photon fusion, induces an azimuthal correlation between the outgoing protons [2]. In practice, if protons are not measured, one has to also include low-mass diffractive excitation in the final state. A review of the experimental situation at the LHC has been given at this conference [5]. Recently, the experimental proof of principle has been given by $\mathrm{CDF}$ at the Tevatron, where exclusive charmonium production has been measured for the first time in hadronic collisions at high energy [6].

With respect to other possible hadronic exchange mechanisms, such as the conjectured Odderon [7], the photon exchange stands out through the very sharp forward peaking induced by the photon pole. Then the relevant photon virtualities are small enough to limit ourselves to quasireal, Weizsäcker-Williams photons. We are dealing with predominantly peripheral collisions, and can expect the effect of absorptive corrections to be only on the $\sim 10 \div 20 \%$-level. The diagrams fig 1 can then be calculated essentially from the amplitude of vector-meson photoproduction on a nucleon. The latter is strongly constrained by data obtained at HERA. In fig. 目 our results for the total photoproduction cross section of $\Upsilon$ are shown against recent data from the ZEUS collaboration (from [8]). For a specific choice of the vector meson wavefunction the agreement is quite good. The calculation is based on a pQCD modelling of Pomeron exchange along the lines of [9], for more details, see [3].

In fig. 3 we show rapidity distributions for various vector mesons in proton-proton (or proton antiproton) collisions. We also show the recent $\mathrm{CDF}$ data [6], the agreement being quite reasonable. It is interesting to observe that the effect of absorptive corrections varies over phase space. Bornlevel results are shown by the dashed lines, and the full (including absorptive corrections) results by the solid lines. Absorption is a stronger effect at larger rapidities. This is related to the fact that there a larger longitudinal momentum transfer is involved, and hence the collision is less pripheral, and therefore absorption is stronger. It is important to keep this in mind if one wants to draw conclusions on the energy dependence of the $\gamma p \rightarrow V p$ photoproduction amplitude from the shape of the rapidity distribution. As far as the production of radially excited vector mesons is concerned, they will be produced in roughly the same ratio as in the photoproduction on the nucleon target.

Let us now turn to the $Z^{0}$ production [4]. Here there are some subtleties in the calculation of the photoproduction amplitude, due to the fact the amplitude has a cut stemming from the $Z^{0} \rightarrow q \bar{q}$ 

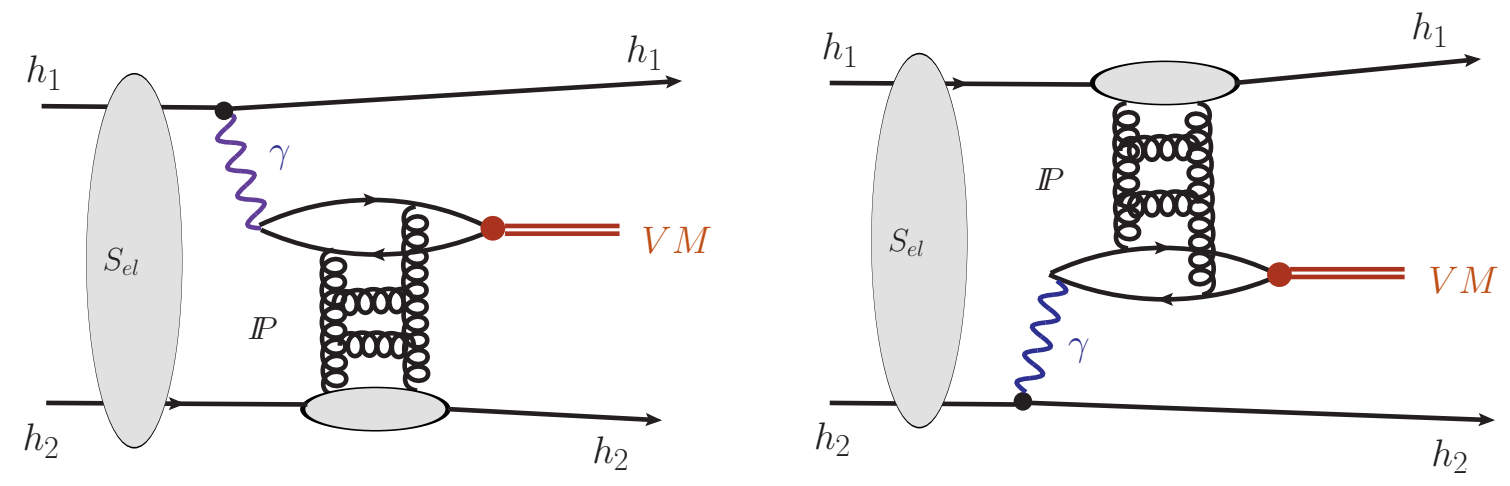

Figure 1: The photon-Pomeron fusion mechanism. The Pomeron exchange is modelled by a pQCD gluon ladder. Also shown is the absorptive correction, including elastic rescattering.
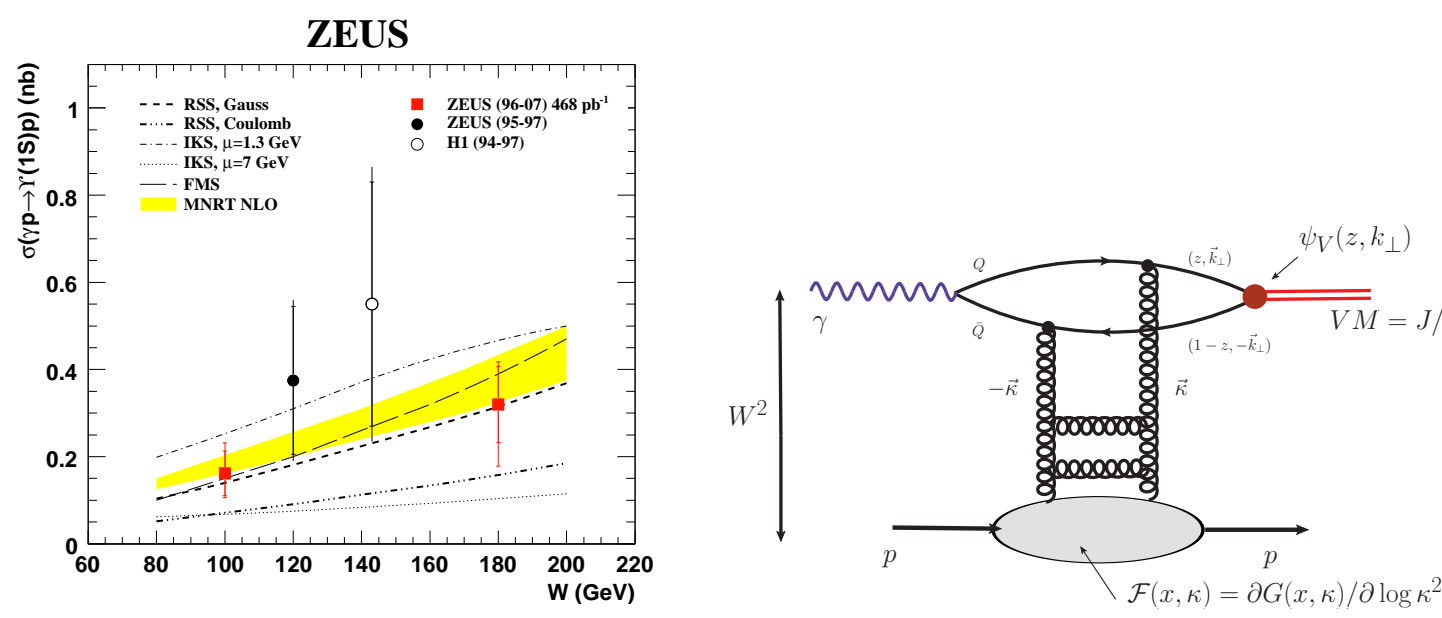

Figure 2: Left: the total cross section for $\gamma p \rightarrow \Upsilon p$ taken from [8]. Our results are labelled RSS. Right: the pQCD model for the production amplitude. The input unintegrated gluon distribution is constrained by HERA data, the vector meson wavefunction has to reproduce the leptonic decay width.

transition, differently from the vector meson case. The photoproduction cross section at HERA energies is exceedingly small, indeed this process has never been observed. In fig. 4 we show rapidity distributions of $Z^{0}$ in $p \bar{p}$ and $p p$ collisions. The thin solid line shows the Born level results, while for the red curves absorption has been included. Absorption effects are stronger than for vector meson production: the heavier $Z^{0}$ boson is produced in substantially less peripheral collisions. The cross section is however disppointingly small. A recent search by CDF [10] came up with an upper limit for the total cross section of $\sim 1 \mathrm{pb}$, roughly a factor thousand bigger than our result.

In summary, cross sections for exclusive vector mesons are of measurable size. The fair agreement of our predictions with Tevatron data suggests that absorption is under reasonable control. A possible measurement at the LHC promises access to the gluon distribution at very small $x$, beyond the HERA domain. 

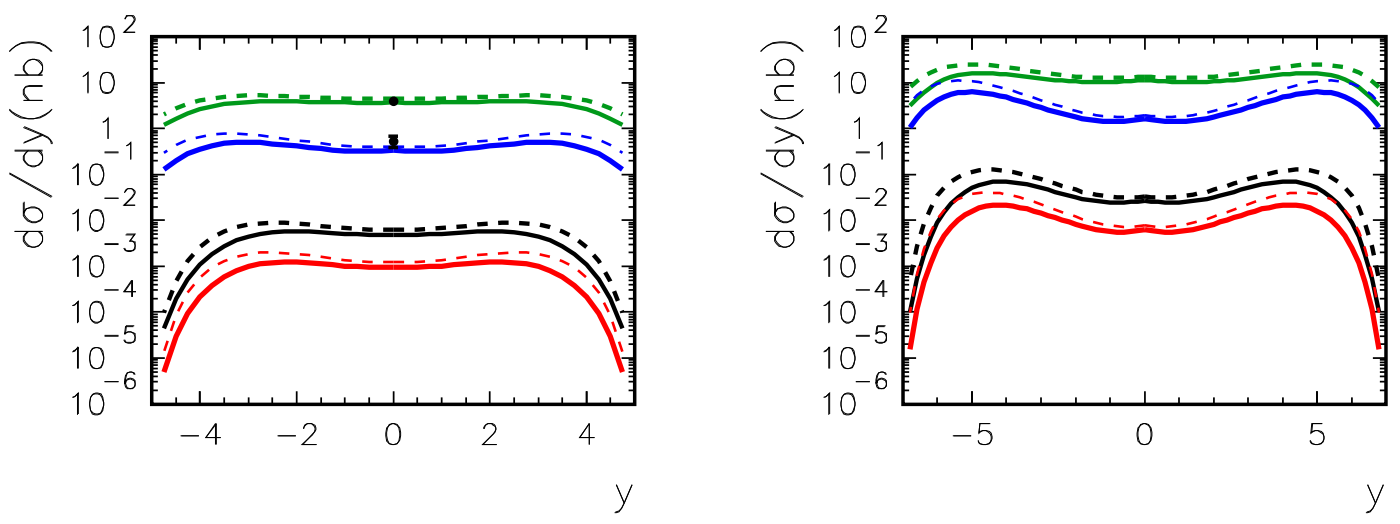

Figure 3: Rapidity spectrum of exclusive vector mesons for Tevatron, $\sqrt{s}=1960 \mathrm{GeV}$, (left) and LHC, $\sqrt{s}=14 \mathrm{TeV}$, (right) energies. Green: $J / \psi$, Blue: $\psi^{\prime}(2 S)$, Black: $\Upsilon$, Red: $\Upsilon(2 S)$. Notice that at LHC energies for $\Upsilon$ at $y \sim 0$ we probe the glue at $x \sim 10^{-3} \div 10^{-4}$, while at $y \sim 5$ effectively $x \sim 10^{-5} \div 10^{-6}$. The data points are from [6]. Dashed lines are without, solid lines with absorption.
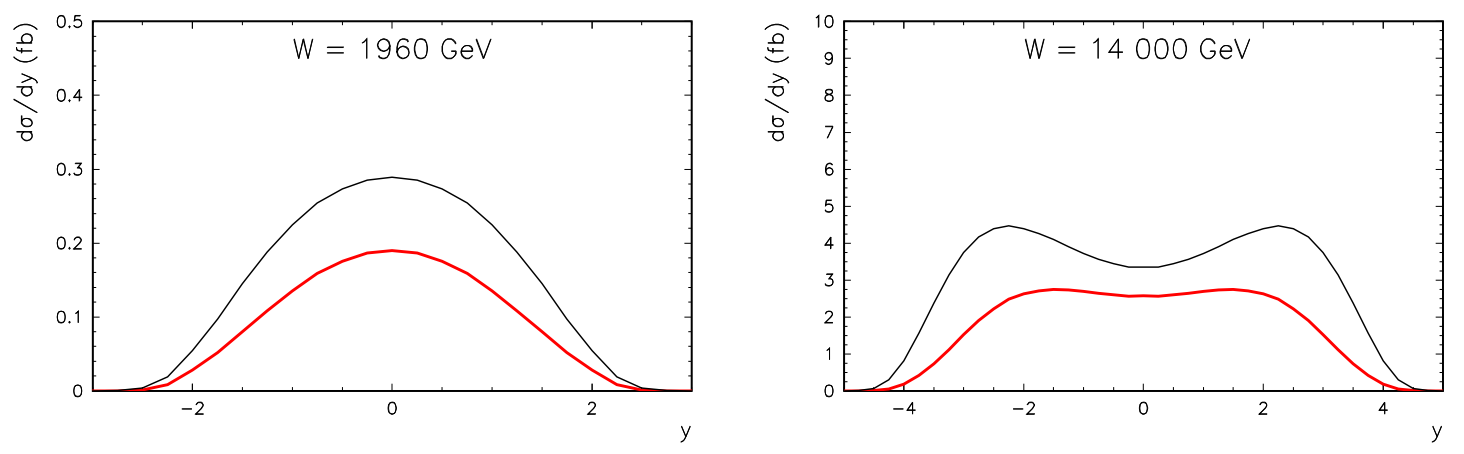

Figure 4: Rapidity spectrum of exclusive $Z^{0}$ 's at Tevatron (left) and LHC (right) energies. The thin black lines are without, the thick red lines with absorption.

\section{References}

[1] A. D. Martin, M. G. Ryskin and V. A. Khoze, "Forward Physics at the LHC," arXiv:0903.2980 [hep-ph]; A. Szczurek, “Central exclusive production at high energies," arXiv:0904.1504 [hep-ph].

[2] W. Schäfer and A. Szczurek, Phys. Rev. D 76 (2007) 094014 [arXiv:0705.2887 [hep-ph]].

[3] A. Rybarska, W. Schäfer and A. Szczurek, Phys. Lett. B 668 (2008) 126 [arXiv:0805.0717 [hep-ph]].

[4] A. Cisek, W. Schäfer and A. Szczurek, arXiv:0906.1739 [hep-ph].

[5] G. Brona, "Mini review, hard diffraction and CEP at LHC", a talk at this conference.

[6] T. Aaltonen et al. [CDF Collaboration], Phys. Rev. Lett. 102 (2009) 242001 [arXiv:0902.1271 [hep-ex]].

[7] A. Schäfer, L. Mankiewicz and O. Nachtmann, Phys. Lett. B 272 (1991) 419. 
[8] [ZEUS Collaboration], “Exclusive photoproduction of $\Upsilon$ mesons at HERA,” arXiv:0903.4205 [hep-ex].

[9] I. P. Ivanov, N. N. Nikolaev and A. A. Savin, Phys. Part. Nucl. 37 (2006) 1 [arXiv:hep-ph/0501034].

[10] T. Aaltonen et al. [CDF Collaboration], Phys. Rev. Lett. 102 (2009) 222002 [arXiv:0902.2816 [hep-ex]]. 